\title{
Electrical and Mechanical Activity in the Human Lower Esophageal Sphincter during Diaphragmatic Contraction
}

\author{
Ravinder K. Mittal, Dudley F. Rochester, and Richard W. McCallum \\ Department of Internal Medicine, University of Virginia, Charlottesville, Virginia 22908
}

\begin{abstract}
To determine the effect of contraction of the diaphragm on the lower esophageal sphincter (LES) pressure, we studied eight healthy volunteers during spontaneous breathing, maximal inspiration, and graded inspiratory efforts against a closed airway (Muller's maneuver). Electrical activity of the crural diaphragm (DEMG) was recorded from bipolar esophageal electrodes, transdiaphragmatic pressure (Pdi) was calculated as the difference between gastric and esophageal pressures, and LES pressure was recorded using a sleeve device. During spontaneous breathing, phasic inspiratory DEMG was accompanied by phasic increases in Pdi and LES pressure. With maximal inspiration, DEMG increased 15-20-fold compared with spontaneous inspiration, and LES pressure rose from an endexpiratory pressure of 21 to $90 \mathrm{mmHg}$. Similar values were obtained during maximal Muller's maneuvers. LES pressure fell promptly when the diaphragm relaxed. Graded Muller's maneuver resulted in proportional increases in the Pdi, LES pressure, and DEMG. The LES pressure was always greater than Pdi and correlated with it in a linear fashion $(P<0.001)$. We conclude that the contraction of the diaphragm exerts a sphincteric action at the LES, and that this effect is an important component of the antireflux barrier.
\end{abstract}

\section{Introduction}

The tonic activity of the smooth muscles of the lower esophageal sphincter (LES) ${ }^{1}$ generates resting LES pressure (1), which is considered to be the major antireflux barrier (2). Interestingly, the sling fibers from the right crus of the diaphragm are arranged in such a way that they could also exert a sphincteric action at the LES (3). Recent studies suggest that the respiration-induced LES pressure oscillations observed in the cat LES are primarily the result of active diaphragmatic contraction (4). Whether the diaphragmatic contraction could actually enhance the LES pressure in the human, and what the physiological significance of such an action might be, are not clear.

In a given skeletal muscle, electrical activity precedes its mechanical activity (5). If the diaphragm does exert a sphinc-

Address reprint requests to Dr. Mittal, Box 145, Department of Internal Medicine, University of Virginia, Charlottesville, VA 22908.

Received for publication 26 February 1987 and in revised form 2 November 1987

1. Abbreviations used in this paper: DEMG, diaphramatic electromyogram; ECG, electrocardiogram; LES, lower esophageal sphincter; Pdi, transdiaphragmatic pressure; TLC, total lung capacity.

J. Clin. Invest.

(C) The American Society for Clinical Investigation, Inc. $0021-9738 / 88 / 04 / 1182 / 08 \quad \$ 2.00$

Volume 81, April 1988, 1182-1189 teric action at the LES, then contraction of the diaphragm should increase its electrical activity, transdiaphragmatic pressure (Pdi), and intraluminal LES pressure. The purpose of our study was to test this hypothesis by recording the simultaneous diaphragmatic electromyogram (DEMG) and LES pressure during spontaneous and voluntary diaphragmatic contraction in humans.

\section{Methods}

\section{Use of the Dent sleeve device to record LES pressure} during diaphragmatic contraction

The LES pressure can be recorded using either side-hole manometry or a Dent sleeve device (6). The former has a disadvantage because normally the LES moves passively up and down with the diaphragm during inspiration and expiration (7). Because the side hole of the manometric catheter remains stationary, the relative axial movement of the LES and side-hole results in a motion artifact. The Dent sleeve device (Fig. 1) takes into account this axial movement, and is more suitable for continuous pressure monitoring of the relatively mobile LES. However, the disadvantage of the sleeve device is its response rate, which is a function of the actual position of the LES along its length (6). The response rate of the sleeve is quick at its proximal end but becomes increasingly slower as the position of the LES moves towards the distal end. We reasoned that if the LES is positioned at the proximal end of the sleeve device at rest (end-expiration), then during diaphragmatic contraction it will move towards its distal end.

To detect the effect of diaphragmatic contraction on the LES pressure, the duration of the diaphragmatic contraction has to be longer than the response rate of the sleeve device at its distal end (8). Therefore, we performed an in vitro study with the sleeve that was used for our experiments (obtained from Arndorfer Medical Specialty, Inc., Milwaukee, WI). The study was performed using a pressure chamber, as used by Dent (6). The entire sleeve device, along with the side holes above and below the sleeve, were positioned in the pressure chamber and each catheter was perfused at a rate of $0.6 \mathrm{ml} / \mathrm{min}$ (rate used for standard manometry). The pressure in the chamber was controlled manually by injecting air into the chamber. The chamber pressure was faithfully recorded by the side holes as well as the sleeve device (Fig. 2). The response rate of the side holes and sleeve device was quick, a pressure rise of $150 \mathrm{mmHg}$ being recorded in $<1 \mathrm{~s}$. The catheter was then placed in the chamber in such a fashion so that only the most distal $1 \mathrm{~cm}$ of the sleeve lay in the chamber, which was then rapidly pressurized to $150 \mathrm{mmHg}$. The pressure recorded by the sleeve increased linearly from baseline to $150 \mathrm{mmHg}$, requiring $17 \mathrm{~s}$ to reach this plateau at the sleeve perfusion rate of $0.6 \mathrm{ml} / \mathrm{min}$. In contrast, the fall in the pressure was recorded simultaneous to the chamber at the proximal as well as the distal end of the sleeve device (Fig. 2).

The dynamic effect of the movement of the pressure zone from proximal to the distal end of the sleeve was then evaluated. The entire sleeve was first placed in the chamber. The chamber pressure was raised and maintained at $150 \mathrm{mmHg}$, and then the catheter was quickly withdrawn from the chamber in such a fashion that only the distal $1 \mathrm{~cm}$ of the sleeve lay in the chamber. The sudden movement of the sleeve resulted in an initial fall of the pressure recorded by the sleeve. The pressure then gradually increased in linear fashion until it plateaued at the chamber pressure (Fig. 3). 

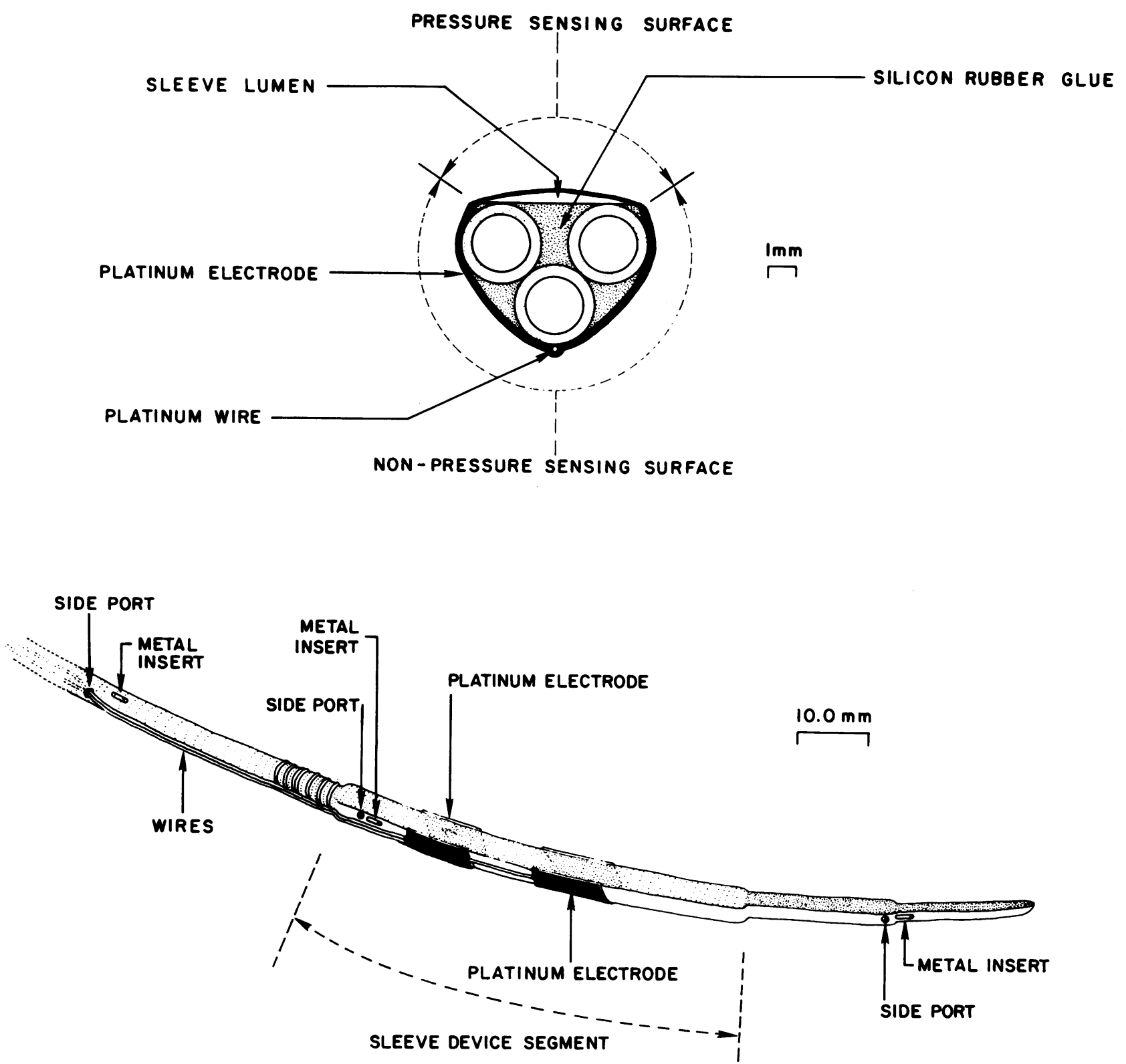

Figure 1. Design of the catheter to record simultaneous LES pressure and DEMG. Two platinum foils of $1 \mathrm{~cm}^{2}$ in surface area are glued to the non-pressure sensing surface of the sleeve device. Two platinum wires are welded to the platinum foils on one end, and externalized at the proximal end of the catheter to connect to the electromyogram amplifier.

The sleeve response rate is also a function of the rate of perfusion, and we determined that, at a rate of perfusion of $1.0 \mathrm{ml} / \mathrm{min}$, only $8 \mathrm{~s}$ was required to achieve $150 \mathrm{mmHg}$ pressure at the distal end of the sleeve. This higher rate of perfusion does not affect the accuracy of pressure measurement (9). For these reasons the voluntary diaphragmatic contractions in our experiments were sustained for more than 8 seconds or until the LES pressure tracing showed a stable and horizontal pattern.

Design of the catheter to record simultaneous LES pressure and diaphragmatic electrical activity. Electrical activity of the crural portion of the diaphragm (DEMG) can be recorded using bipolar electrodes placed close to the esophagogastric junction (10-13). The DEMG recorded through intraesophageal electrodes correlates with and is comparable to activity recorded by electrodes implanted directly into the crural part of the diaphragm $(14,15)$.
The sleeve device has an almost tubular structure and its anatomy is such that only one quarter of its circumference is utilized as a pressure sensing surface and the remaining three quarters serve as a supporting structure (Fig. 1). Two thin platinum foil squares, $0.05 \mathrm{~mm}$ thick and $1 \mathrm{~cm}^{2}$ in surface area (Thomas Scientific, Cherry Hill, NJ) were glued to the non-pressure sensing surface of the sleeve device. These electrodes were placed $1 \mathrm{~cm}$ apart, and the proximal one was spaced $1 \mathrm{~cm}$ from the proximal margin of the sleeve device. Two teflon-coated, insulated platinum wires were guided through one of the side holes of the manometric catheter (Fig. 1) to be externalized at the proximal end of the catheter. At their distal ends, the insulation was removed up to $0.5 \mathrm{~cm}$ from the tips, and the wires were arc-welded to the under surface of the platinum foils.

The arrangement of the electrodes is important since the best DEMG is recorded when the electrodes are located as close to the 


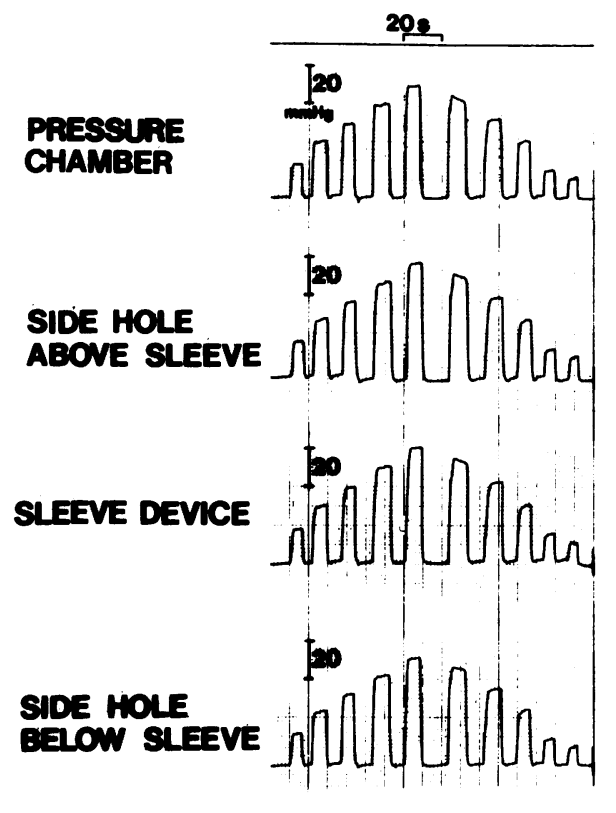

Figure 2. Validation of the pressure measurement by the side hole and the sleeve device. The catheter, with the entire sleeve device and with side holes above and below the sleeve was placed in the pressure chamber. The pressure in the chamber was manually controlled by injecting air in the chamber. Note that the chamber pressure was recorded faithfully by the side holes as well as the sleeve device. Also, the response rate of the sleeve device was quick and identical to that of the side holes.

diaphragm as possible $(14,15)$. With the proximal margin of sleeve in the LES, these electrodes should be very close to the diaphragm because the diaphragm encircles the distal part of the LES (16).

The signals for the DEMG were amplified through a Sensormedics model 9852 amplifier (Sensormedics, Anaheim, CA). The low and high frequency filter cutoffs were 40 and $1,000 \mathrm{~Hz}$, respectively. The output from the amplifier was recorded and also fed into a movingtime average filter using $200 \mathrm{~ms}$ as the time-averaging periods for quantitation of the DEMG (17). The outputs of the amplified and filtered electromyogram signals, and of the pressure amplifiers, were recorded on an eight-channel recorder (Sensormedics).

Subjects. The study was performed in eight healthy human volunteers. Their mean age was 27 yr (range 19-38). The group consisted of five men and three women. All subjects denied any history of illness and were not taking any medications. Also, all of these subjects were found to have normal esophageal motility. The protocol for the study was approved by the Human Investigation Committee at the University of Virginia, and all subjects signed an informed consent prior to entering the study.

\section{Experimental design}

All studies were performed after a 6-h fast, with the subjects in a supine position. The specially designed catheter was passed through the nose into the esophagus and stomach. The whole length of the sleeve device was positioned first into the stomach to obtain gastric pressure. The catheter was then withdrawn in a stepwise fashion, $0.5 \mathrm{~cm}$ at a time, until the side hole at the proximal end of the sleeve recorded esophageal pressure just above the LES. With the proximal end of the sleeve straddling the LES, a phasic DEMG was recorded with each inspiration.

In the first part of the experiment recordings were made for $15 \mathrm{~min}$, while the subjects rested. In the second part of the experiment DEMG and the pressures in the esophagus, LES, and stomach were monitored

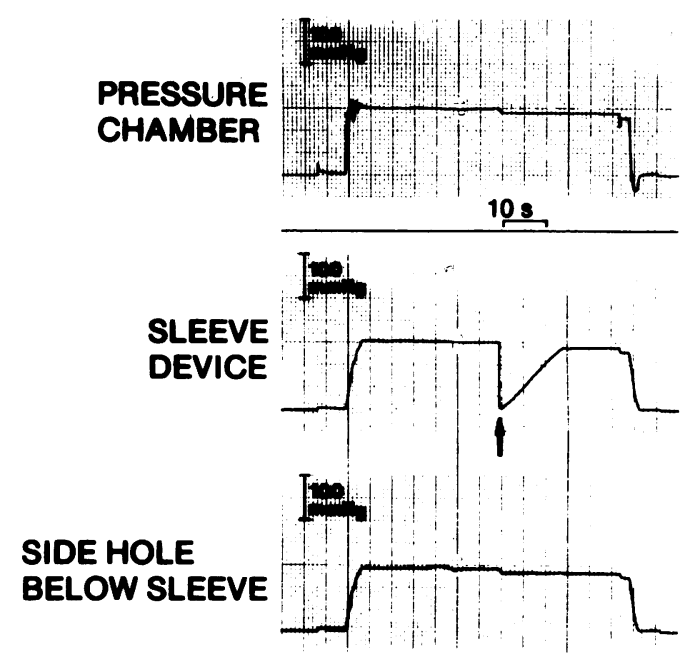

Figure 3. Effect of movement of the high-pressure zone on the sleeve device. The entire sleeve was first placed in the chamber, which was pressurized to $150 \mathrm{mmHg}$. The catheter was then suddenly withdrawn from the chamber (marked by arrow) in such a fashion so that only the distal $1 \mathrm{~cm}$ of the sleeve lay in the chamber. Note that movement of the sleeve results in a rapid initial drop in the recorded pressure. Recorded pressure then gradually increases until it reaches the chamber pressure, at which point the tracing shows a horizontal pattern. Also, note that when the chamber pressure is released, the fall in pressure as recorded by the sleeve is quick and almost simultaneous with the fall in chamber pressure.

during three time intervals: time 1 , end-tidal expiration; time 2 , subjects inspired to total lung capacity (TLC), and time 3, relax against a closed airway. During the inspiration maneuver, subjects maintained an open airway so that they had to sustain diaphragmatic contraction. Subjects maintained this state for $8 \mathrm{~s}$ or until the LES pressure tracing showed a stable and horizontal pattern. At the onset of time 3, while still at TLC, the subjects were instructed to relax against a closed glottis, which allows the diaphragm to relax (18).

The third part of the experiment consisted of graded diaphragmatic contractions against a closed airway (Muller's maneuver). In these efforts, the subjects inspired from the full expiratory position (residual volume). The airway was connected to a pressure gauge (Dwyers Instruments, Inc., Michigan City, IN) which recorded negative mouth pressure generated during the inspiratory efforts. The mouth pressure recorded during maximal effort was called $100 \%$ for that particular subject. Using visual feedback from the pressure gauge, subjects were then instructed to generate various fractions of the maximal mouth pressure, ranging from $25 \%$ to $75 \%$ maximal. For each individual, four or five measurements were made for each of the two or three levels of the Muller's maneuver. Each effort was sustained for at least $8 \mathrm{~s}$. Subjects were not allowed to swallow for $30 \mathrm{~s}$ prior to and during any of the maneuvers. If during this time period an esophageal contraction did occur, the maneuver was repeated.

Esophageal and gastric pressures were recorded simultaneously with the side holes located above and below the sleeve device, respectively. The LES and esophageal pressures were measured in reference to the end-expiratory gastric pressure, which was set at zero. The Pdi gradient was calculated as the difference between gastric and esophageal pressures. At end-expiration the Pdi was the pressure difference recorded by the side hole above the sleeve device as it was pulled from the stomach into the esophagus. This Pdi was added to the further changes in Pdi as they occurred during various maneuvers. Since esophageal pressure was always negative in relation to the stomach, the 
Pdi was always a positive number. DEMG was standardized for each individual, and the maximum DEMG during maximal inspiratory effort and maximum Muller's maneuver was taken as $100 \%$ for that individual.

The data are presented as mean \pm SEM and were analyzed using paired $t$ tests and linear regression. A difference was considered significant when $P<0.05$.

\section{Results}

Pressure and electrical activity in the LES at rest. With the proximal end of sleeve positioned in the LES, three patterns of phasic electrical activity were recorded from the electrodes that were placed on the sleeve device: (a) electrocardiogram (ECG); (b) DEMG with each respiration; and $(c)$ a spike burst with each esophageal contraction. The three patterns could be easily differentiated from one another based on their morphological appearance and relationship with ECG recorded from the chest leads, respiration, and manometrically recorded esophageal contraction. No other electrical activity could be recognized in the windows between these three patterns of electrical activity (Fig. 4).

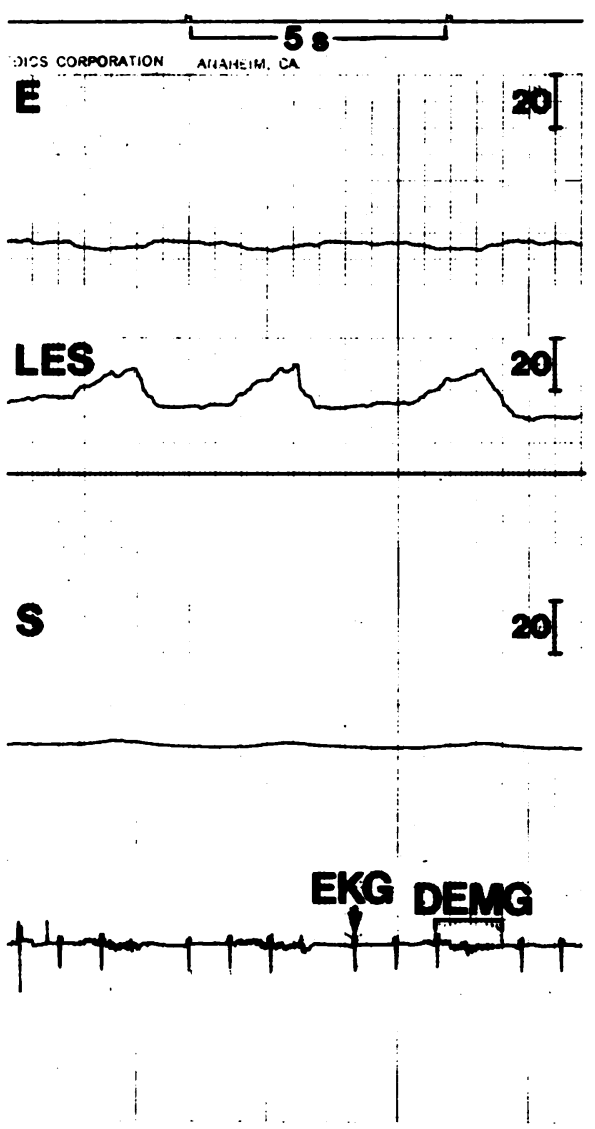

Figure 4. A recording of LES pressure and electrical activity of the heart (EKG) and diaphragm (DEMG). The DEMG occurred during each spontaneous inspiration. Note the rise and fall in LES pressure corresponds to the onset and cessation of each DEMG. Horizontal line at the bottom of the LES tracing corresponds with gastric pressure ( $E$, esophagus; $S$, stomach).
There was a marked variability in the resting end-expiratory LES pressure, both in the same individual and among different subjects. The LES pressure in most individuals varied from 10 to $40 \mathrm{mmHg}$. In spite of this tonic LES activity, no electrical activity was recorded that could be attributed to the spike potentials originating in the LES. The DEMG amplitude was 5-15 $\mu \mathrm{V}$ and occurred with each spontaneous inspiration (phasic DEMG). Accompanying each phasic DEMG there was an increase in the LES pressure. The magnitude of this increase ranged from 10 to $25 \mathrm{mmHg}$. The Pdi during end-expiration ranged from 4 to $6 \mathrm{mmHg}$. With each spontaneous inspiration, the esophageal pressure became more negative by 4-5 $\mathrm{mmHg}$ and the gastric pressure increased by $2-3 \mathrm{mmHg}$. Therefore, the Pdi during spontaneous inspirations ranged from 8 to $11 \mathrm{mmHg}$ (Fig. 4).
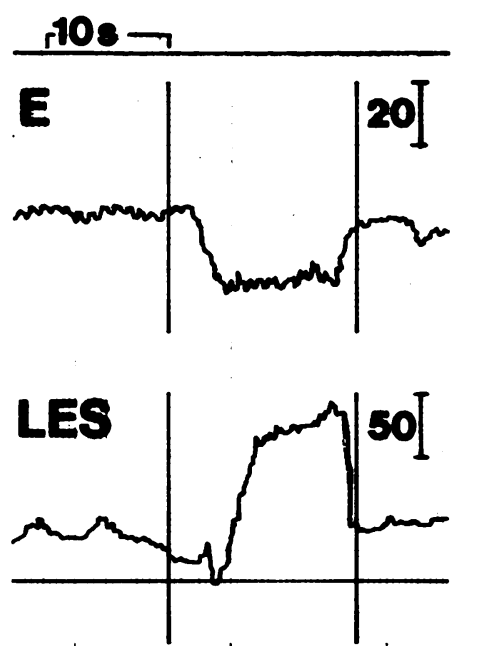

Figure 5. Recording of the esophageal $(E)$, LES, and stomach $(S)$ pressures along with DEMG during three time periods: time 1, end-expiration; time 2 , subject inspired to total lung capacity and kept the airway open (sustained diaphragmatic contraction); time 3 , while still at the total lung capacity subject relaxed against a closed glottis (diaphragmatic relaxation). During time 2, note the sustained DEMG, and the LES pressure which initially fell but then rose gradually until it reached a peak and stabilized. The time course of the LES pressure increase is related to movement of the LES and the sleeve response time (see text). The LES pressure and DEMG fell rapidly and simultaneously at the onset of time 3. The pressure remained negative and positive in the esophageal and stomach respectively during time 2 and there was equalization of these pressures in time 3 . The horizontal line at the bottom of the LES tracing corresponds with gastric pressure. (DEMG 1 is a recording in the direct mode; DEMG 2 is the same signal as DEMG 1 that is rectified and computed through a 200-ms moving time average filter.) 
LES pressure and electrical activity during maximal inspiratory effort with airway open and closed. In every individual a maximal inspiratory effort to TLC resulted in a 15-20fold increase of DEMG, as compared with spontaneous inspiration (Fig. 5, time 2). The amplitude of DEMG was sustained as long as the airway was kept open. With relaxation against a closed airway (Fig. 5, time 3), there was an instantaneous drop in DEMG to a level that was only $10-15 \%$ of the maximal inspiratory effort. This incomplete relaxation of the diaphragm during closure of the airway is consistent with the observation of Miranda and Lourenco (18).

Throughout time 2 the esophageal pressure remained negative and there was an increase in the gastric pressure. Thus, Pdi increased abruptly from $4.8 \pm 0.5 \mathrm{mmHg}$ in time 1 to $32 \pm 3.8$ $\mathrm{mmHg}$ in time 2. However, the increase in LES pressure was always 2-2.5 times greater than the increase in Pdi (Fig. 6). There was a net increase of LES pressure from 21.2 \pm 3.0 $\mathrm{mmHg}$ in time 1 to $89 \pm 12.0 \mathrm{mmHg}$ in time 2. Even though the amplitudes of DEMG and Pdi remained constant, LES pressure recorded in time 2 increased slowly, because of the response rate of the Dent sleeve. 4-6 s after the onset of sustained effort the LES tracing showed a horizontal pattern (Fig. 5).

During time 3 there was an equalization of the esophageal and gastric pressures, resulting in fall in Pdi to $4.9 \pm 1.8 \mathrm{mmHg}$. Along with this, there was a simultaneous drop in the LES pressure. The LES pressure in time 3 was not significantly different from that in time 1 (Fig. 6).

LES pressure and DEMG during graded diaphragmatic contraction. Each Muller's maneuver resulted in a negative mouth pressure, a negative esophageal pressure, a positive Pdi, and an increase in the LES pressure (Fig. 7). The effect of Muller's maneuver on the gastric pressure was variable. In four individuals there was a small increase ranging from 5 to 10 $\mathrm{mmHg}$. In two, there was no change and in the remaining two the gastric pressure actually became negative. This is consistent with the result of DeTroyer and Estenne (19).

With the onset of a partial or maximal inspiratory effort, DEMG and Pdi abruptly increased, and esophageal pressure

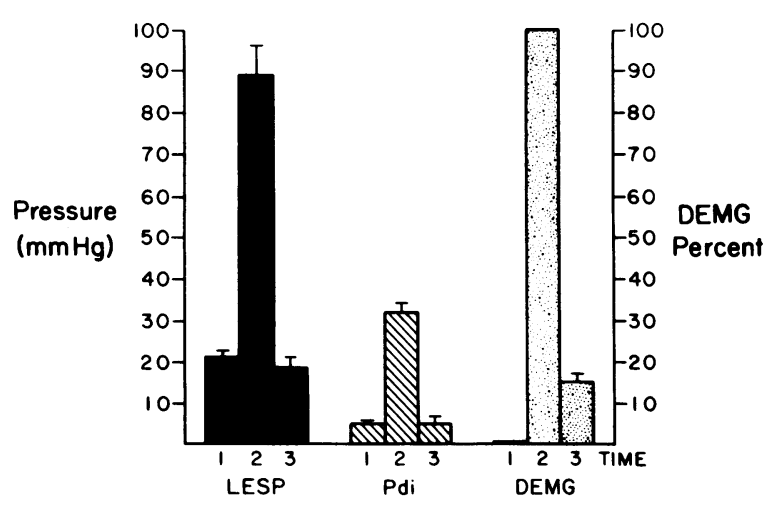

Figure 6. The LES pressure, Pdi, and DEMG in eight subjects during three time periods: time 1, end-expiration; time 2, subjects inspired to TLC and kept the airway open (sustained diaphragmatic contraction); time 3, at TLC subjects closed the airway (diaphragm relaxed). The DEMG at $100 \%$ was $15-18$ times larger than the DEMG during spontaneous respiration among various subjects.
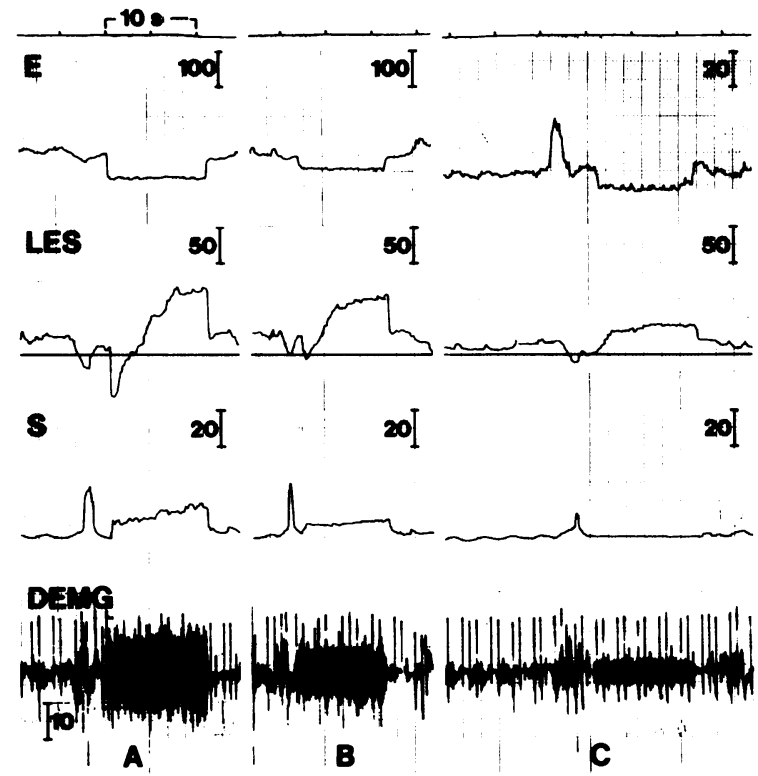

Figure 7. Esophageal $(E)$, LES, and stomach $(S)$ pressures along with DEMG recording in one subject during graded Muller's maneuvers. Horizontal line at the bottom of LES tracing corresponds with gastric pressure. Each maneuver resulted in a negative esophageal pressure, an increase in LES pressure, and an increase in DEMG. $(A) 100 \%$ or maximal inspiratory effort; $(B) 66 \%$ effort; $(C) 33 \%$ effort. The esophageal pressure, LES pressure, and DEMG during $66 \%$ and $33 \%$ were proportionately smaller than the $100 \%$ effort. The rise in LES pressure relative to the DEMG was slow at the onset but both the parameters fell rapidly and simultaneously at the end of each maneuver.

abruptly decreased. Thereafter, these variables remained at constant levels. The recorded increase in LES pressure was again gradual, approaching a plateau level in linear fashion. Once the plateau pressure (true LES pressure) had been reached, DEMG, Pdi, and LES pressure remained constant until completion of the Muller's maneuver, at which point both electrical activity and pressures fell rapidly and simultaneously.

During submaximal and maximal Muller's efforts, LES pressure, Pdi, and DEMG increased in proportion (Figs. 7 and 8). The pressures recorded during three levels of Muller's maneuver in one subject are shown in Table I. The relationships between mouth pressure, Pdi, LES pressure, and DEMG were linear in each individual (Fig. 8, $A-C$ ). When DEMG, LES pressure, Pdi, and mouth pressure were expressed as \% maximum, the slopes and intercepts of DEMG, LES pressure and Pdi as functions of mouth pressure are nearly identical (Fig. 8 $D$ ). There was a strong linear correlation between the LES pressure and Pdi for all subjects (Fig. 9, data points). Also, the slopes of LES pressure-Pdi relationships in the eight individual subjects were fairly constant (Fig. 9, solid lines).

\section{Discussion}

Our data show that in healthy subjects, the LES pressure during diaphragmatic contraction is substantially higher than the end-expiratory LES pressure. The rise in LES pressure is proportional to the force of diaphragmatic contraction which was 

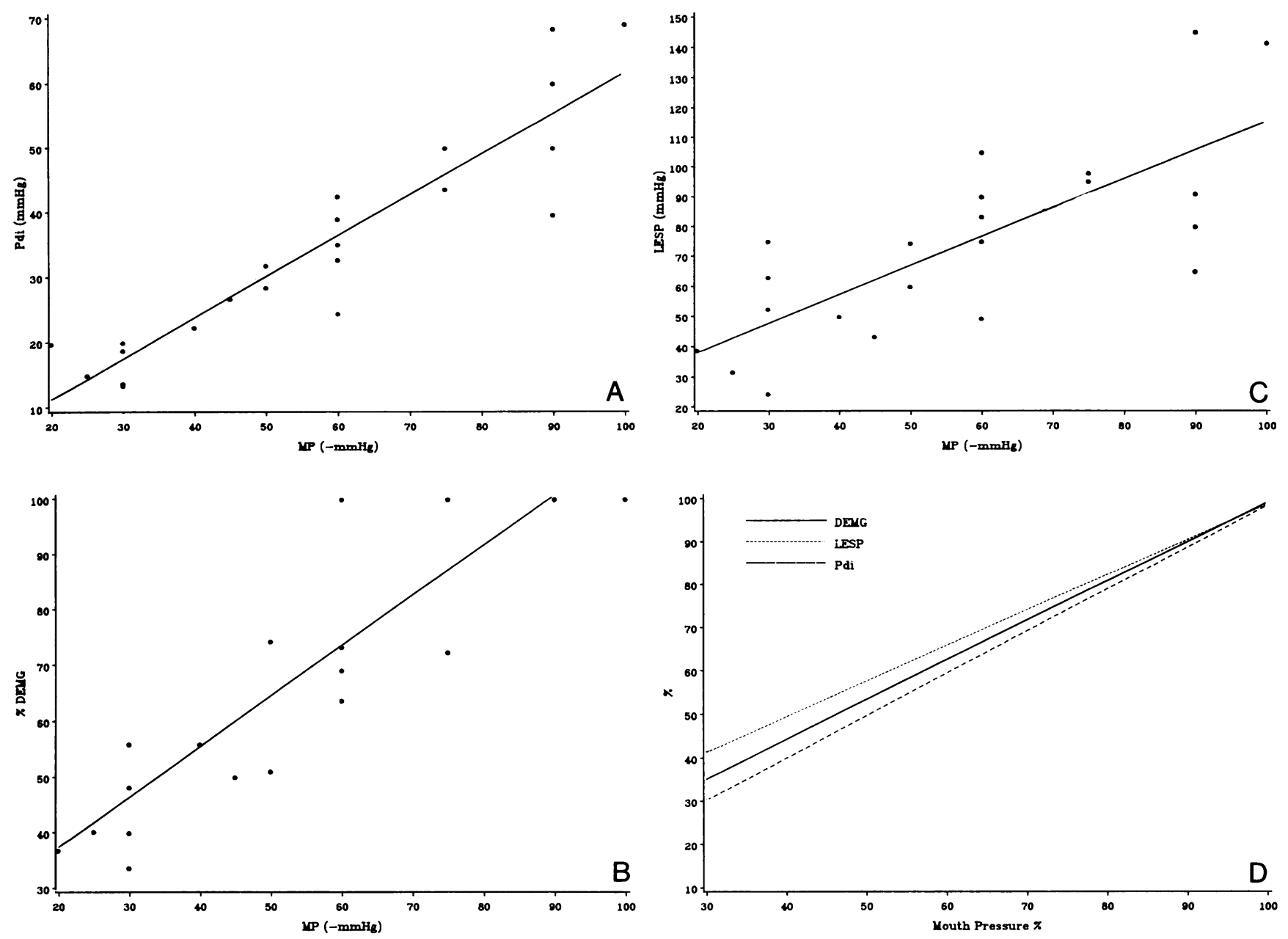

Figure 8. Relationship between mouth pressure $(M P)$ and $(A)$ Pdi gradient in $\mathrm{mmHg} ;(B)$ percent diaphragmatic electric activity (DEMG); (C) LES pressure (LESP) in mmHg during graded Muller's maneuver. Each data point represents the mean of four to five measurements in one individual. The actual mouth pressure correlated in a linear fashion to the Pdi $(r=0.92)$, percent DEMG $(r=0.89)$, and the LES pressure $(r=0.76)$. (D) The relationship among LES pressure, Pdi, and DEMG during graded Muller's maneuver plotted

estimated from Pdi and diaphragmatic electrical activity (DEMG). We have been able to record DEMG along with LES pressure using intraesophageal electrodes, and we show that DEMG, Pdi, and LES pressure increase in a linear fashion.

Table I. Data in One Subject During Graded Muller's Maneuvers

\begin{tabular}{cccc}
\hline Effort level & Pdi & LES pressure & DEMG \\
\hline$\%$ & $m m H g$ & $m m H g$ & arbitrary units \\
100 & $69 \pm 2.0$ & $142 \pm 10.6$ & $14.5 \pm 0.8$ \\
75 & $44 \pm 1.0$ & $98 \pm 4.6$ & $10.5 \pm 0.8$ \\
50 & $29 \pm 1.4$ & $75 \pm 4.0$ & $7.4 \pm 0.7$ \\
0 & $4.0 \pm 0$ & $25 \pm 2.4$ & $0 \pm 0$
\end{tabular}

Values given as mean $\pm \mathrm{SEM}$.

against the mouth pressure. The LES pressure, Pdi, DEMG, and mouth pressure during maximal inspiratory effort was taken as $100 \%$ for each individual. Subject then performed two or three fractions of the maximal effort (from $25 \%$ to $75 \%$ ). Each line was plotted from the mean of the data in eight individuals at various percent mouth pressure. There was strong linear correlation between the percent mouth pressure and the percent LES pressure $(r=0.94)$, Pdi $(r$ $=0.96)$, and DEMG $(r=0.98)$.

The increase in LES pressure is always greater than the increase in Pdi. Cessation of the diaphragmatic contraction results in a sudden fall in LES pressure.

The pinch-cock effect of the diaphragm on the esophagus can be easily observed during endoscopy, but whether a similar effect occurs during manometric LES pressure recordings has been controversial. The difficulty stems from the fact that the LES moves passively along with the diaphragm $(20,21)$, whereas the manometric catheter remains stationary. This relative axial movement of the LES and the side hole of the manometric catheter results in a motion artifact in the LES pressure recordings. The LES pressure during diaphragmatic contraction can be precisely and accurately measured by the sleeve device, but the rate at which recorded LES pressure increases is delayed, owing to the response rate of the sleeve (Fig. 3). We think that actually during diaphragmatic contraction the LES pressure rises as rapidly as DEMG and Pdi and 


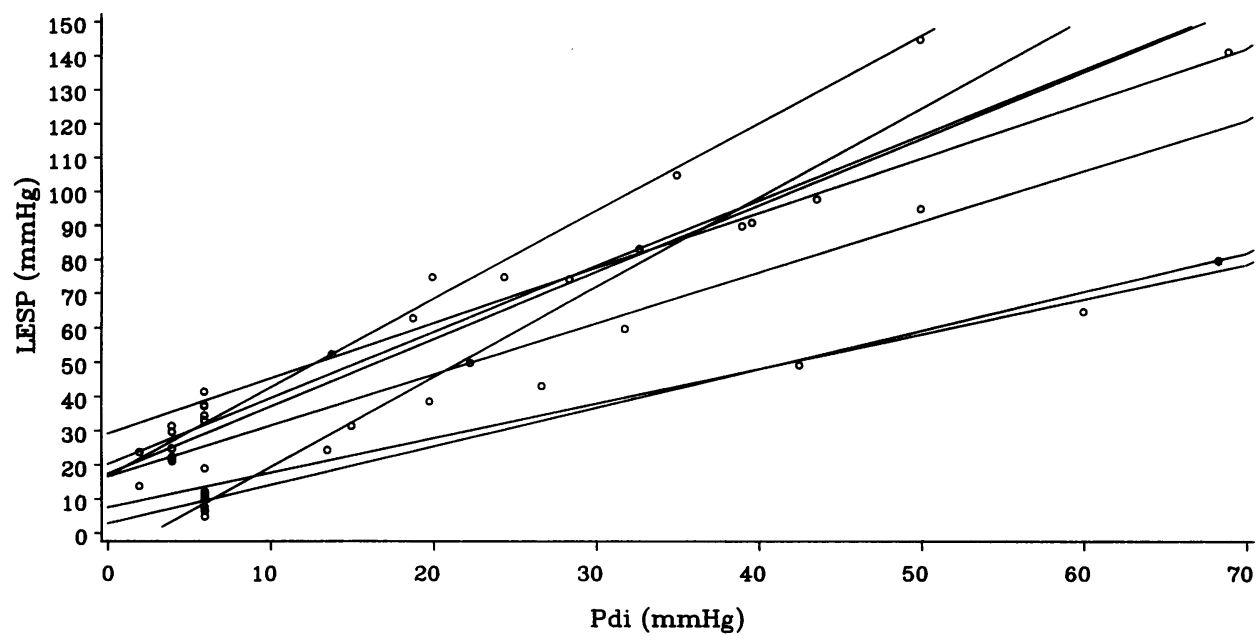

Figure 9. Relationship between Pdi and LES pressure (LESP). Each line represents the data from one individual and was plotted from four sets of data points which were $(a)$ with the mouth pressure of 0 or end-expiratory pressure or $(b) 100 \%$ inspiratory effort and then two sets of readings from the graded effort. Each data point represents the mean of four to five measurements. In each individual the Pdi correlated strongly with the LES pressure (ranging from 0.94 to 0.99 ) and notice that the slope of lines in eight individuals was fairly constant (ranging from 1.01 to 2.62 ). the apparent slow rise in LES pressure in our recording is artifactual. The rapid fall of LES pressure in association with the DEMG at the end of each maneuver suggests a strong temporal relationship between the two events. Similar to ours, the studies by Welch and Gray (16) and Boyle et al. (4) show that the LES pressure increase during spontaneous inspiration is primarily the result of a sphincterlike action of the active diaphragmatic contraction.

The absence of electrical activity in the windows between DEMG and ECG suggests that tonic LES contraction in humans is not related to electrical spike activity. Other investigators have reported that, in cats and opossums, a considerable proportion of the LES tone is the result of electrical spike activity originating in the smooth muscles of the $\operatorname{LES}(22,23)$. In our recordings, we could not identify any spike activity that could be correlated with the LES tone. In the windows between DEMG and ECG, the only spike activity that could be recognized was the one that associated with the esophageal contraction. The absence of electrical spike activity in our study may be due to the following: $(a)$ a species difference (23); (b) our method of recording, because we used electrodes that were placed axially (other investigators have suggested that spike activity in the circular muscle is best recorded by the transversely placed electrodes [22]); (c) we recorded electrical activity only between 40 and $1,000 \mathrm{~Hz}$ because DEMG is best recorded between the above frequencies, whereas other investigators recorded spike activity using a frequency band between DC and $30 \mathrm{~Hz}(22)$. It is possible that spike activity originating in the LES is best recorded in the lower frequency range. However, it is also possible that the LES tone in the human is totally spike independent because, clearly, even in animal studies, a significant proportion of the LES pressure is spike independent (23).

Could the electrical activity that we recorded in the LES during diaphragmatic contraction be originating in the smooth muscle of the LES rather than the diaphragmatic skeletal muscles? A number of arguments would make it seem highly unlikely: (a) The typical frequency of LES spike is $20-50 / \mathrm{min}$ $(0.3-0.8 \mathrm{~Hz})(22)$, and we recorded frequencies above $40 \mathrm{~Hz}$. The high frequency of electrical spike activity recorded as DEMG is characteristic of diaphragmatic motor unit firing $(24,25)$. (b) Animal studies reveal that DEMG recorded by intraesophageal electrodes placed at the esophagogastric junction and directly implanted wires in the diaphragm are comparable $(13,14)$ and we found strong correlation between the amplitude of DEMG and depth of inspiration in each individual. (c) In the absence of diaphragmatic contraction, no electrical spike activity was recognized even in the presence of tonic LES pressure. (d) In an individual when a LES pressure of $40 \mathrm{mmHg}$ was recorded in the absence of diaphragmatic contraction (during phasic LES pressure changes), there was no electrical activity in the LES, whereas in the same individual when $40 \mathrm{mmHg}$ LES pressure was observed in the presence of diaphragmatic contraction, electrical activity was present. (e) The rapid rise and fall of LES pressure at the onset and completion of the diaphragmatic contraction is characteristic of skeletal rather than smooth muscle activity.

Our study clearly suggests that diaphragmatic contraction has a sphincteric action at the esophagogastric junction and the crural diaphragm may be thought of as an external lower esophageal sphincter. What is the physiologic significance of such an action? The Pdi in fact represents the gastroesophageal pressure gradient, and it can be considered a driving force for the gastric content to reflux into the esophagus. The LES pressure, on the other hand, is a barrier against gastroesophageal reflux. In a resting end-expiratory state, the Pdi is only of the magnitude of 4-5 mmHg. Therefore, an LES pressure of 5 $\mathrm{mmHg}$ or more should provide an effective antireflux barrier. The observation by Dent et al. (26) that acid reflux into the esophagus does not occur unless an end-expiratory LES pressure falls to $<5 \mathrm{mmHg}$ suggests that an intrinsic LES pressure of $5 \mathrm{mmHg}$ is a sufficient antireflux barrier under resting conditions, and any more pressure is only a reserve (27).

During diaphragmatic contraction, the Pdi can be in the range of $30-70 \mathrm{mmHg}$ or more (28), considerably higher than the end expiratory LES pressure. Our study shows that it is during this period that the LES pressure is augmented. In a given individual the LES pressure increase is always higher than the Pdi, and the two increase in a linear fashion. It is reasonable to propose that, in order to prevent gastroesophageal reflux, the ventilatory function of the diaphragm that requires the generation of Pdi should be coupled to lower esophageal sphincteric function. Indeed, we found that under physiologic conditions the circumferential pressure generated in the 
LES by diaphragmatic contraction always exceeds the axial pressure that is represented by Pdi. We suggest that the sphincteric action of the crural diaphragm is an important component of the antireflux barrier and its dysfunction may contribute to gastroesophageal reflux.

\section{Acknowledgments}

Authors are indebted to Joyce Dolan and Mary Beth MeachumWhitehill for typing this manuscript, Donald Kaiser for assistance in statistical analysis and data presentation, and G. Annette Whelchel for technical assistance.

This work was supported in part by a research grant from American College of Gastroenterology.

\section{References}

1. Goyal, R. K., and S. Rattan. 1976. Genesis of basal sphincter pressure: effect of tetrodotoxin on LES pressure in opossum in vivo. Gastroenterology. 61:62-67.

2. Richter, J., and D. O. Castell. 1982. Gastroesophageal reflux. Pathogenesis, diagnosis, and therapy. Ann. Intern. Med. 97:93-103.

3. Clemeute, C. D. 1985. The diaphragm. In Gray's Anatomy. Thirtieth American Edition. Lea \& Febiger, Philadelphia. 479-480.

4. Boyle, J. T., S. M. Altschuler, T. E. Nixon, D. N. Tuckman, A. I. Pack, and S. Cohen. 1985. Role of the diaphragm in the genesis of lower esophageal sphincter pressure in the cat. Gastroenterology. 88:723-730.

5. Guyton, P. 1981. Contraction of skeletal muscle. In Textbook of Medical Physiology. W. B. Saunders Co., Philadelphia. 122-137.

6. Dent, J. 1976. A new technique for continuous sphincter pressure measurement. Gastroenterology. 71:263-267.

7. Dodds, W. J., E. T. Stewart, W. J. Hogan, J. J. Steff, and R. C. Arndorfer. 1974. Effect of esophageal movement on intraluminal esophageal pressure recordings. Gastroenterology. 67:592-600.

8. Dodds, W. J., J. J. Steff, and W. J. Hogan. 1976. Factors determining pressure measurement accuracy by intraluminal manometry. Gastroenterology. 70:117-123.

9. Linehan, J. H., J. Dent, W. J. Dodds, and W. J. Hogan. 1985. Sleeve device functions as a starling resistor to record sphincter pressure. Am. J. Physiol. 248:G251-255.

10. Draper, M., P. Ladefoged, and D. Whitteridge. 1959. Respiratory muscle in speech. J. Speech Res. 2:16-27.

11. Lourenco, R. V., M. S. Cherniack, J. R. Malm, and A. P. Fishman. 1966. Nervous output from the respiratory center during obstructed breathing. J. Appl. Physiol. 21:527-533.

12. Agostoni, E., G. Sant Ambrogio, and H. P. Carrasco. 1960. Electromyography of the diaphragm in man and transdiaphragmatic pressure. J. Appl. Physiol. 15:1093-1097.
13. Petit, M., G. Milic-Emili, and L. Delhez. 1960. Role of the diaphragm in breathing in conscious normal man: an electromyographic study. J. Appl. Physiol. 15:1101-1106.

14. Grassino, A. E., W. A. Whitelaw, and J. Milic-Emili. 1976. Influence of lung volume and electrode position on electromyography of the diaphragm. J. Appl. Physiol. 40:971-975.

15. Kim, M. J., W. S. Driz, J. Danon, W. Machnach, and J. T. Sharp. 1978. Effects of lung volume and electrode position on esophageal diaphragm EMG. J. Appl. Physiol. 45:392-398.

16. Welch, R. W. and J. E. Gray. 1982. Influence of respiration on recording of LES pressure in humans. Gastroenterology. 83:590-594.

17. Hill, A. R., D. L. Kaiser, and D. F. Rochester. 1984. Effect of thoracic volume and shape on electromechanical coupling in abdominal muscles. J. Appl. Physiol. 56:1294-1301.

18. Miranda, J. M., and R. V. Lourenco. 1968. Influence of diaphragm activity on the measurement of total chest compliance. $J$. Appl. Physiol. 24:741-746.

19. DeTroyer, A., and M. Estenne. 1981. Limitation of measurement of transdiaphragmatic pressure in detecting diaphragmatic weakness. Thorax. 36:169-174.

20. Dodds, W. J., E. T. Stewart, D. Hodges, and F. F. Zboralske. 1973. Movement of the feline esophagus associated with respiration and peristalsis. An evaluation using tantalum markers. J. Clin. Invest. 52:1-13.

21. Winans, C. S. 1972. Alteration of lower esophageal sphincter characteristics with respiration and proximal esophageal balloon distension. Gastroenterology. 62:380-388.

22. Asoh, R., and R. K. Goyal. 1978. Electrical activity of the opossum lower esophageal sphincter in vivo. Gastroenterology. 74:835-840.

23. Goyal, R. K., and B. W. Cobb. 1981. Motility of the pharynx, esophagus, and esophageal sphincters. In Physiology of the Gastrointestinal Tract. L. R. Johnson, editor. Raven Press, New York. 379.

24. Schweitzer, T. W., J. W. Fitzgerald, J. A. Bowden, and P. Lynne-Davies. 1979. Spectral analysis of human inspiratory diaphragmatic electromyograms. J. Appl. Physiol. 46:152-165.

25. Bishop, B., S. Settle, and J. Hersch. 1981. Single motor unit activity in the diaphragm of cat during pressure breathing. J. Appl. Physiol. 50:348-357.

26. Dent, J., W. J. Dodds, R. H. Friedman, T. Seriguchi, W. J. Hogan, R. C. Arndorfer, and D. J. Petrie. 1980. Mechanism of gastroesophageal reflux in recumbent asymptomatic human subjects. J. Clin. Invest. 65:256-267.

27. Dodds, W. J., J. Dent, W. J. Hogan, J. F. Helm, R. H. Hauswer, G. K. Patel, and M. S. Eqide. 1982. Mechanism of gastroesophageal reflux in patients with reflux esophagitis. N. Engl. J. Med. 307:15471552.

28. Bellemare, F., B. B. Ritchie, and J. J. Woods. 1986. Contractile properties of the human diaphragm in vivo. J. Appl. Physiol. 61:1153-1161. 